\title{
The connection between the Galactic halo and ancient Dwarf Satellites
}

\author{
Emanuele Spitoni* \\ Dipartimento di Fisica, Università di Trieste \\ E-mail: spitoni@oats.inaf.it
}

\section{Fiorenzo Vincenzo}

Dipartimento di Fisica, Università di Trieste

E-mail: vincenzodoats.inaf.it

\section{Francesca Matteucci}

Dipartimento di Fisica, Università di Trieste

E-mail: matteuccidoats. inaf.it

\section{Donatella Romano}

I.N.A.F. Osservatorio Astronomico di Bologna

E-mail: donatella.romanodoabo.inaf.it

\begin{abstract}
We explore the hypothesis that the classical and ultra-faint dwarf spheroidal satellites of the Milky Way have been the building blocks of the Galactic halo by comparing their $[\mathrm{O} / \mathrm{Fe}]$ and $[\mathrm{Ba} / \mathrm{Fe}]$ versus $[\mathrm{Fe} / \mathrm{H}]$ patterns with the ones observed in Galactic halo stars. Oxygen abundances deviate substantially from the observed abundances in the Galactic halo stars for $[\mathrm{Fe} / \mathrm{H}]$ values larger than -2 dex, while they overlap for lower metallicities. On the other hand, for the $[\mathrm{Ba} / \mathrm{Fe}]$ ratio the discrepancy is extended at all $[\mathrm{Fe} / \mathrm{H}]$ values, suggesting that the majority of stars in the halo are likely to have been formed in situ. Therefore, we suggest that $[\mathrm{Ba} / \mathrm{Fe}]$ ratios are a better diagnostic than $[\mathrm{O} / \mathrm{Fe}]$ ratios. Moreover, we show the effects of an enriched infall of gas with the same chemical abundances as the matter ejected and/or stripped from dwarf satellites of the Milky Way on the chemical evolution of the Galactic halo. We find that the resulting chemical abundances of the halo stars depend on the assumed infall time scale, and the presence of a threshold in the gas for star formation.
\end{abstract}

Frontier Research in Astrophysics - II

23-28 May 2016

Mondello (Palermo), Italy

\footnotetext{
* Speaker.
} 


\section{Introduction}

According to the $\Lambda \mathrm{CDM}$ paradigm, a Milky Way-like galaxy must have formed by the merging of a large number of smaller systems. In particular, dwarf spheroidal galaxies (dSphs) were proposed in the past as the best candidate small progenitor systems, which merged through cosmic time to eventually form the stellar halo component of the Galaxy (e.g. Grebel 2005).

On the other hand, Fiorentino et al. (2015) using RR Lyrae stars as tracers of the Galactic halo ancient stellar component, showed that dSphs do not appear to be the major building-blocks of the halo. Leading physical arguments suggest an extreme upper limit of 50\% to their contribution.

In recent years, the Sloan Digital Sky Survey (SDSS, York et al. 2000) were able to discover an entirely new population of hitherto unknown stellar systems: the so-called ultra faint dwarf spheroidal galaxies (UfDs), which are characterized by extremely low luminosities, high dark matter content, and very old and iron-poor stellar populations (Belokurov et al. 2006; Norris et al (2008, 2010); Brown et al 2012). The number of UfDs has increased constantly in the last decade and completeness estimates suggest that many more of these faint satellites are still to be discovered in the Local Group (Tollerud et al. 2008). This fact might place them as the survived building blocks of the Galaxy stellar halo, dramatically lacking in the past.

In Spitoni et al. (2016) we test the hypothesis that dSph and UfD galaxies have been the building blocks of the Galactic halo, by assuming that the halo formed by accretion of stars belonging to these galaxies. Moreover, extending the results of Spitoni (2015) to detailed chemical evolution models in which the IRA is relaxed, we explored the scenario, in which the Galactic halo formed by accretion of chemically enriched gas originating from dSph and UfD galaxies.

\section{The chemical evolution models}

\subsection{The Milky Way}

We consider the following two reference chemical evolution models for the MW galaxy:

1. The classical two-infall model (2IM) presented by Brusadin et al. (2013). The Galaxy is assumed to have formed by means of two main infall episodes: the first formed the halo and the thick disc, the second the thin disc.

2. The two-infall model plus outflow of Brusadin et al. (2013; here we indicate it as the 2IMW model). In this model a gas outflow occuring during the halo phase with a rate proportional to the star formation rate through a free parameter is considered. Following Hartwick (1976), the outflow rate is defined as:

$$
\frac{d \sigma_{w}}{d t}=-\omega \psi(t)
$$

where $\omega$ is the outflow efficiency.

In Table 1 the principal characteristics of the two chemical evolution models for the MW are summarized: in the second column the time-scale $\tau_{H}$ of halo formation, in the third the time-scale $\tau_{D}$ of the thin disc formation, are drawn. The adopted threshold in the surface gas density for the star formation (SF) is reported in columns 4. In column 5 the exponent of the Schmidt (1959) law 

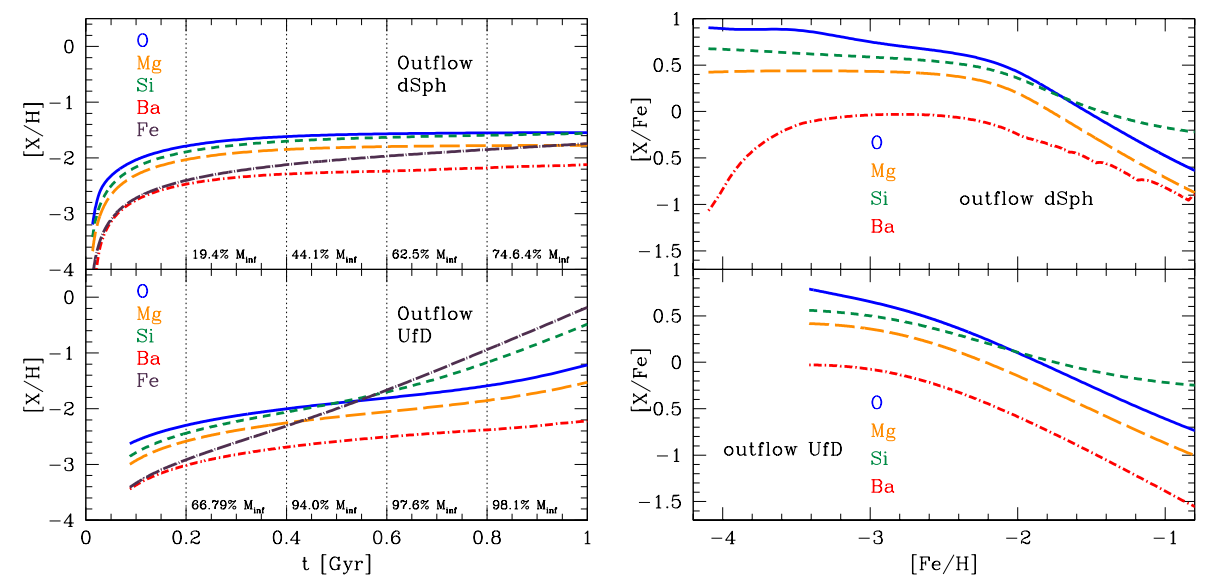

Figure 1: Left panels: The evolution in time of the chemical abundances for $\mathrm{O}, \mathrm{Mg}, \mathrm{Si}, \mathrm{Ba}, \mathrm{Fe}$ in the gas ejected as galactic wind from dSphs and UfDs. The onset of the wind happens at later times compared with $\mathrm{dSph}$ objects. We also indicate the cumulative ejected gas mass by outflows at $0.2,0.4,0.6$, and $0.8 \mathrm{Gyr}$ in terms of percentage of the infall mass $M_{\text {inf }}$. Right panels: The abundance ratio $[\mathrm{X} / \mathrm{Fe}]$ as a function of $[\mathrm{Fe} / \mathrm{H}]$ for the following chemical elements: $\mathrm{O}, \mathrm{Mg}, \mathrm{Si}$, and $\mathrm{Ba}$ of the outflowing gas ejected by a dSph galaxy, and by a UfD galaxy.

The Milky Way: the solar neighborhood model parameters

\begin{tabular}{cccccccc} 
Models & $\begin{array}{c}\tau_{H} \\
{[\mathrm{Gyr}]}\end{array}$ & $\begin{array}{c}\tau_{D} \\
{[\mathrm{Gyr}]}\end{array}$ & $\begin{array}{c}\text { Threshold } \\
{\left[\mathrm{M}_{\odot} \mathrm{pc}^{-2}\right]}\end{array}$ & $k$ & $\begin{array}{c}v \\
{\left[\mathrm{Gyr}^{-1}\right]}\end{array}$ & IMF & $\begin{array}{c}\omega \\
{\left[\mathrm{Gyr}^{-1}\right]}\end{array}$ \\
\hline 2IM & 0.8 & 7 & $\begin{array}{c}\text { (halo-thick disc) } \\
7 \text { (thin disc) }\end{array}$ & 1.5 & $\begin{array}{c}\text { (halo-thick disc) } \\
1 \text { (thin disc) }\end{array}$ & Scalo (1986) & $/$ \\
\hline 2IMW & 0.2 & 7 & $\begin{array}{c}4 \text { (halo-thick disc) } \\
7 \text { (thin disc) }\end{array}$ & 1.5 & $\begin{array}{c}\text { 2(halo-thick disc) } \\
1 \text { (thin disc) }\end{array}$ & Scalo (1986) & 14 \\
\hline
\end{tabular}

Table 1: Parameters of the chemical evolution models for the Milky Way (Spitoni al. 2016) in the solar neighborhood.

is shown, in columns 6 and 7 we report the star formation efficiency and the IMF, respectively. Finally, in the last column the presence of the wind is indicated in term of the efficiency $\omega$.

\subsection{The dSph and UfD galaxies}

In Table 2 the main parameters of generic models for "classical" dSph and UfD galaxies are reported, respectively. The star formation efficiency $v$, the exponent $k$ of the Kennicutt (1998) law, and the wind efficiency $\omega$ are drawn in column one, two and three, respectively. In the other columns are reported: the infall timescale (column 4); total infall gas mass (column 5); mass of the dark matter halo (column 6); effective radius of the luminous (baryonic) matter (column 7); ratio between the core radius of the DM halo and the effective radius of the luminous matter (column 8); in column 9 the adopted IMF is indicated. We assume that UfD objects are characterized by a very small star formation efficiency $\left(0.01 \mathrm{Gyr}^{-1}\right)$ and by an extremely short time scale of formation 


\begin{tabular}{cccccccccc}
\hline Models & $\begin{array}{c}v \\
{\left[\mathrm{Gyr}^{-1}\right]}\end{array}$ & $k$ & $\omega$ & $\begin{array}{c}\tau_{\text {inf }} \\
{[\mathrm{Gyr}]}\end{array}$ & $\begin{array}{c}M_{\text {inf }} \\
{\left[M_{\odot}\right]}\end{array}$ & $\begin{array}{c}M_{D M} \\
{\left[M_{\odot}\right]}\end{array}$ & $\begin{array}{c}r_{L} \\
{[\mathrm{pc}]}\end{array}$ & $S=\frac{r_{L}}{r_{D M}}$ & IMF \\
\hline dSphs & 0.1 & 1 & 10 & 0.5 & $10^{7}$ & $3.4 \cdot 10^{8}$ & 260 & 0.52 & Salpeter(1955) \\
Ufds & 0.01 & 1 & 10 & 0.001 & $10^{5}$ & $10^{6}$ & 35 & 0.1 & Salpeter (1955) \\
\hline
\end{tabular}

Table 2: Parameters of the chemical evolution model for general dSph and UfD galaxies (Spitoni et al. 2016).

(0.001 Gyr). We point out that in the modeling the dSphs and UfDs we did not consider any threshold in the gas density for star formation, as in Vincenzo et al. (2014).

The time at which the galactic wind starts in $\mathrm{dSphs}$ is at $0.013 \mathrm{Gyr}$ after the galactic formation, whereas for UfDs at $0.088 \mathrm{Gyr}$. As expected, the UfD galaxies develop a wind at later times because of the smaller adopted star formation efficiency (SFE).

\subsection{Nucleosynthesis prescriptions}

We adopt the nucleosynthesis prescriptions of Romano et al. (2010, model 15), who provide a compilation of stellar yields able to reproduce several chemical abundance patterns in the solar neighborhood. In particular, they assume the following sets of stellar yields.

For barium, we assume the stellar yields of Cescutti et al. (2006, model 1, table 4). In particular, Cescutti et al. (2006) includes the metallicity-dependent stellar yields of $\mathrm{Ba}$ as computed by Busso et al. (2001), in which barium is produced by low-mass AGB stars, with mass in the range $1.0 \leq M \leq 3.0 \mathrm{M}_{\odot}$, as an s-process neutron capture element. A second channel for the $\mathrm{Ba}-$ production was included by Cescutti et al. (2006), by assuming that massive stars in their final explosive stage are capable of synthesizing $\mathrm{Ba}$ as a primary r-process element. Such r-process Ba producers have mass in the range $12 \leq M \leq 30 \mathrm{M}_{\odot}$.

We remark on the fact that the contribution to barium from massive stars was empirically computed by Cescutti et al. (2006), by matching the $[\mathrm{Ba} / \mathrm{Fe}]$ versus $[\mathrm{Fe} / \mathrm{H}]$ abundance pattern as observed in the Galactic halo stars. They assumed for massive stars the iron stellar yields of Woosley \& Weaver (1995), as corrected by François et al. (2004).

\section{The enriched infall of gas}

The novelty of Spitoni et al. (2016) is to take into account in a self-consistent way time dependent abundances. The gas infall law is the same as in the 2IM or 2IMW models and it is only considered a time dependent chemical composition of the infall gas mass.

We take into account the enriched infall from $\mathrm{dSph}$ and UfD galaxies predicted by the following 2 models:

- Model i): The infall of gas which forms the Galactic halo is considered primordial up to the time at which the galactic wind in dSphs (or UfDs) starts. After this moment, the infalling gas presents the chemical abundances of the wind. In Figs. 2 and 3 we refer to this model with the label "Name of the reference model+dSph"or "Name of the reference model+UfD". 
- Model ii): we explore the case of a diluted infall of gas during the MW halo phase. In particular, after the galactic wind develops in the dSph (or UfD) galaxy, the infalling gas has a chemical composition which, by 50 per cent, is contributed by the dSph (or UfD) outflows; the remaining 50 per cent is contributed by primordial gas of a different extra-galactic origin. In all the successive figures and in the text, we refer to these models with the labels "Name of the MW model+dSph (or UfD) MIX".

In the two left panels of Fig. 1, we show the evolution in time of the chemical composition of the outflowing gas from the dSph and the UfD galaxy for $\mathrm{O}, \mathrm{Mg}, \mathrm{Si}, \mathrm{Ba}$ and $\mathrm{Fe}$. It is worth noting that in the outflows from UfD galaxies the $\mathrm{Fe}$ and $\mathrm{Si}$ abundances are larger than in the outflows from dSphs. We recall that $\mathrm{Fe}$ is mostly produced by Type Ia $\mathrm{SNe}$ and $\mathrm{Si}$ is also produced in a non negligible amount by the same SNe. Because in our models the ratio between the time scale of formation between UfD and dSph is extremy low $\left(\tau_{\text {inf }}(\mathrm{UfD}) / \tau_{\text {inf }}(\mathrm{dSph})=2 \times 10^{-3}\right.$, at later times the pollution from Type IA SN is more evident in the UfD outflow. In the two right panels the $[X / \mathrm{Fe}]$ versus $[\mathrm{Fe} / \mathrm{H}]$ abundance patterns are presented, where $X$ corresponds to $\mathrm{O}, \mathrm{Mg}, \mathrm{Si}$, and $\mathrm{Ba}$.

\section{The Results}

In this section, we present the results of our chemical evolution models for the Galactic halo.

\subsection{The Results: the Galactic halo in the model 2IM}

In the left panel of Fig. 2, the predicted $[\mathrm{O} / \mathrm{Fe}]$ versus $[\mathrm{Fe} / \mathrm{H}]$ abundance patterns are compared with the observed data in Galactic halo stars. In order to directly test the hypothesis that Galactic halo stars have been stripped from $\mathrm{dSph}$ or UfD systems, we show the predictions of chemical evolution models for a typical dSph and UfD galaxy (long dashed lines in grey and black, respectively). The two models cannot explain the $[\alpha / \mathrm{Fe}]$ plateau which Galactic halo stars exhibit for $[\mathrm{Fe} / \mathrm{H}] \geq-2.0 \mathrm{dex}$; in fact, halo stars have always larger $[\mathrm{O} / \mathrm{Fe}]$ ratios than dSph and UfD stars.

Moreover, in the left panel of Fig. 2 we show the effects of the enriched infall with chemical abundances taken by the outflowing gas from $\mathrm{dSph}$ and Ufd objects on the $[\mathrm{O} / \mathrm{Fe}]$ versus $[\mathrm{Fe} / \mathrm{H}]$ relation.

First we analyze the results with the enriched infall coming from dSph galaxies. We see that for oxygen we obtain a better agreement with the data in the halo phase when we consider the enriched infall models. We recall that a key ingredient of the 2IM model is the presence of a threshold in the gas density in the star formation (SF) fixed at $4 \mathrm{M}_{\odot} \mathrm{pc}^{-2}$ in the Halo-thick disc phase. During the halo phase such a critical threshold is reached only at $t=0.356 \mathrm{Gyr}$ from the Galaxy formation. On the other hand, when including the environmental effect, we have to consider also the time for the onset of the galactic wind, which in the $\mathrm{dSph}$ model occurs at $t_{\mathrm{gw}}=0.013 \mathrm{Gyr}$.

Therefore, the SF begins after 0.356 Gyr from the beginning of Galaxy formation, and this fact explains the behavior of the curves with enriched infalls in the left panel of Fig. 2: during the first 0.356 Gyr in both " $2 \mathrm{IM}+\mathrm{dSph}$ " and " $2 \mathrm{IM}+\mathrm{dSph}$ MIX" models, no stars are created, and the chemical evolution is traced by the gas accretion. In Figs. 2 and 3 we indicate with thinner lines the ISM chemical evolution phases in which the SFR did not start yet in the Galactic halo, and during which stars are no created. To summarize, for the model " $2 \mathrm{IM}+\mathrm{dSph}$ " we distinguish three different phases in the halo chemical evolution: 

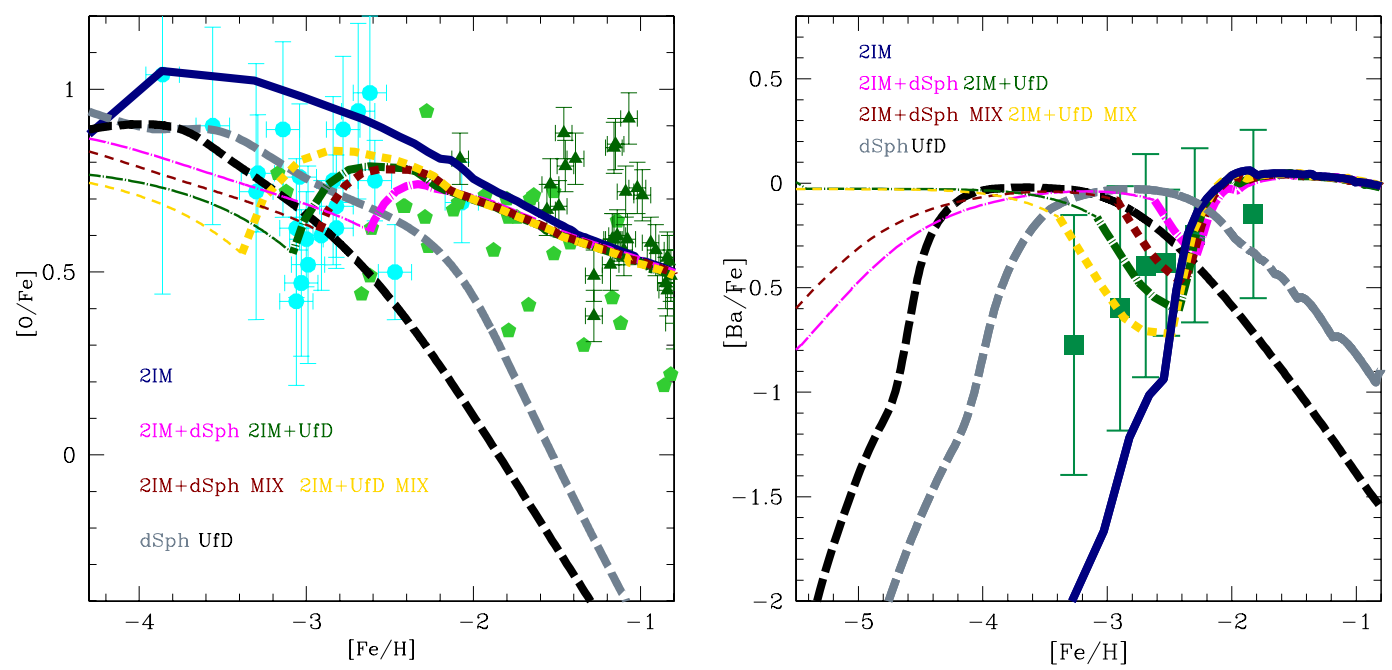

Figure 2: The abundance ratios $[\mathrm{O} / \mathrm{Fe}]$ vs $[\mathrm{Fe} / \mathrm{H}]$ (left panel) and $[\mathrm{Be} / \mathrm{Fe}]$ vs $[\mathrm{Fe} / \mathrm{H}]$ (right panel) in the solar neighborhood for the reference model 2IM are drawn with the solid blue line. Models of the Galactic Halo with the enriched infall from $\mathrm{SSp}$ : the magenta dashed dotted line and the red short dashed line represent the models 2IM+dSph and 2IM+dSph MIX, respectively. Models of the Galactic Halo with the enriched infall from UfDs: the green dashed dotted line and the yellow short dashed line represent the models 2IM+UfD and 2IM+UfD MIX, respectively. Thinner lines indicate the ISM chemical evolution phases in which the SFR did not start yet in the Galactic halo, and during which stars are no created. Models of the dSph and UfD galaxies: The long dashed gray line represents the abundance ratios for the dSph galaxies, whereas long dashed black line for the UfD galaxies. Observational Oxygen data of the Galactic Halo: Cayrel et al. (2004) (cyan circles), Akerman et al. (2004) (light green pentagons), Gratton et al. (2003) (dark green triangles). Observational Barium data of the Galactic Halo: Frebel (2010).

- Phase 1): 0-0.013 Gyr, the infall is primordial, the wind in $\mathrm{dSphs}$ has not started yet, and there is no SF;

- Phase 2): 0.013-0.356 Gyr, the infall is enriched by dSphs, the SFR is zero in this phase;

- Phase 3): 0.356-1 Gyr; the infall is enriched by dSphs, the SFR is different from zero.

During phase 3), the SF takes over, and increases the $[\mathrm{O} / \mathrm{Fe}]$ values because of the pollution from massive stars on short time-scales.

We note that the entire spread of the data cannot be explained assuming a time dependent enriched infall with the same abundances of the outflowing gas from dSph galaxies, even if there is a better agreement with the halo data in comparison to the model with primordial infall.

It is important to underline that, until the SF is non-zero, no stars are created; however, since our models follow the chemical abundances in the gas phase, the solely contribution to the ISM chemical evolution before SF begins is due to the time dependent enriched infall. It means that in the "2IM+dSph" model the first stars that are formed have $[\mathrm{Fe} / \mathrm{H}]$ values larger than -2.4 dex.

In this case, to explain data for stars with $[\mathrm{Fe} / \mathrm{H}]$ smaller than -2.4 dex we need stars formed in dSph systems (see the model curve of the chemical evolution of dSph galaxies). 
Concerning the results with the enriched infall from UfD outflow abundances, we recall here that in our reference model for UfD galaxies, the wind starts at $0.08 \mathrm{Gyr}$. The model results for the halo still reproduce the data but with the same above mentioned caveat. The models with enriched infalls which show the fastest chemical enrichment are the ones with infall abundances taken from the outflows of dSph objects, because the galactic winds occur earlier than in UfD systems.

In Spitoni et al. (2016) we also show the results for $\mathrm{Mg}$ and $\mathrm{Si}$. As concluded for the $[\mathrm{O} / \mathrm{Fe}]$ versus $[\mathrm{Fe} / \mathrm{H}]$ abundance diagram, our reference chemical evolution models for $\mathrm{dSph}$ and UfD galaxies cannot explain the observed Galactic halo data over the entire range of $[\mathrm{Fe} / \mathrm{H}]$ abundances. This rules out the hypothesis that all Galactic halo stars were stripped or accreted in the past from dSphs or UfDs.

In the right panel of Fig. 2, we show the results for the $[\mathrm{Ba} / \mathrm{Fe}]$ versus $[\mathrm{Fe} / \mathrm{H}]$ abundance diagram. The observational data are from Frebel et al. (2010), as selected and binned by Cescutti et al. (2013). By looking at the figure, the 2IM model does not provide a good agreement with the observed data-set for $[\mathrm{Fe} / \mathrm{H}]<-2.5 \mathrm{dex}$. The initial increasing trend of the $[\mathrm{Ba} / \mathrm{Fe}]$ ratios in the 2IM model is due to the contribution of the first Ba-producers, which are massive stars with mass in the range $12-30 \mathrm{M}_{\odot}$.

One can also appreciate that our chemical evolution models for dSphs and UfDs fail in reproducing the observed data, since they predict the $[\mathrm{Ba} / \mathrm{Fe}]$ ratios to increase at much lower $[\mathrm{Fe} / \mathrm{H}]$ abundances than the observed data. That is due to the very low SFEs assumed for dSphs and UfDs, which cause the first Ba-polluters to enrich the ISM at extremely low $[\mathrm{Fe} / \mathrm{H}]$ abundances. The subsequent decrease of the $[\mathrm{Ba} / \mathrm{Fe}]$ ratios is due to the large iron content deposited by Type Ia $\mathrm{SNe}$ in the ISM, which happens at still very low $[\mathrm{Fe} / \mathrm{H}]$ abundances in $\mathrm{dSphs}$ and UfDs. Hence, in the range $-3.5 \leq[\mathrm{Fe} / \mathrm{H}] \leq-2.5 \mathrm{dex}$, while Galactic halo stars exhibit an increasing trend of the $[\mathrm{Ba} / \mathrm{Fe}]$ versus $[\mathrm{Fe} / \mathrm{H}]$ abundance ratio pattern, UfD stars show a decreasing trend (see also Koch et al. 2013).

In the right panel of Fig. 2, all our models involving an enriched infall from dSphs and UfDs deviate substantially from the observed trend of the $[\mathrm{Ba} / \mathrm{Fe}]$ versus $[\mathrm{Fe} / \mathrm{H}]$ abundance pattern in Galactic halo stars. Such a discrepancy enlarges for $[\mathrm{Fe} / \mathrm{H}]<-2.4$ dex, where those models predict always larger $[\mathrm{Ba} / \mathrm{Fe}]$ ratios than the $2 \mathrm{IM}$ model.

\subsection{The Results: the Galactic halo in the model 2IMW}

In this subsection we show the results when the time dependent enriched infall is applied to the reference model 2IMW. In the left panel of Fig. 3 we show the results in terms of $[\mathrm{O} / \mathrm{Fe}]$ versus $[\mathrm{Fe} / \mathrm{H}]$ in the solar neighborhood. In the reference model 2IMW the SFR starts at $0.05 \mathrm{Gyr}$. Comparing model "2IMW+dSph" in the left panel of Fig. 3 with model " $2 \mathrm{IM}+\mathrm{dSph}$ " in the left panel of Fig. 2, we can see that the former shows a shorter phase 2) than the latter.

The model results for the model "2IMW+UfD" in the left panel of Fig. 3 overlap to the reference model 2IMW at almost all [Fe/H] abundances. In fact, since in the UfD galactic model the wind starts at $0.088 \mathrm{Gyr}$ and, at this instant, in the model 2IMW the SF is already active Therefore the effect of the enriched infall is almost negligible compared to the pollution of chemical elements produced by dying halo stars.

Concerning the $[\mathrm{Ba} / \mathrm{Fe}]$ versus $[\mathrm{Fe} / \mathrm{H}]$ abundance pattern, in the right panel of Fig. 3 we compare the predictions of our models with the Galactic halo data. We notice that the 2IMW 

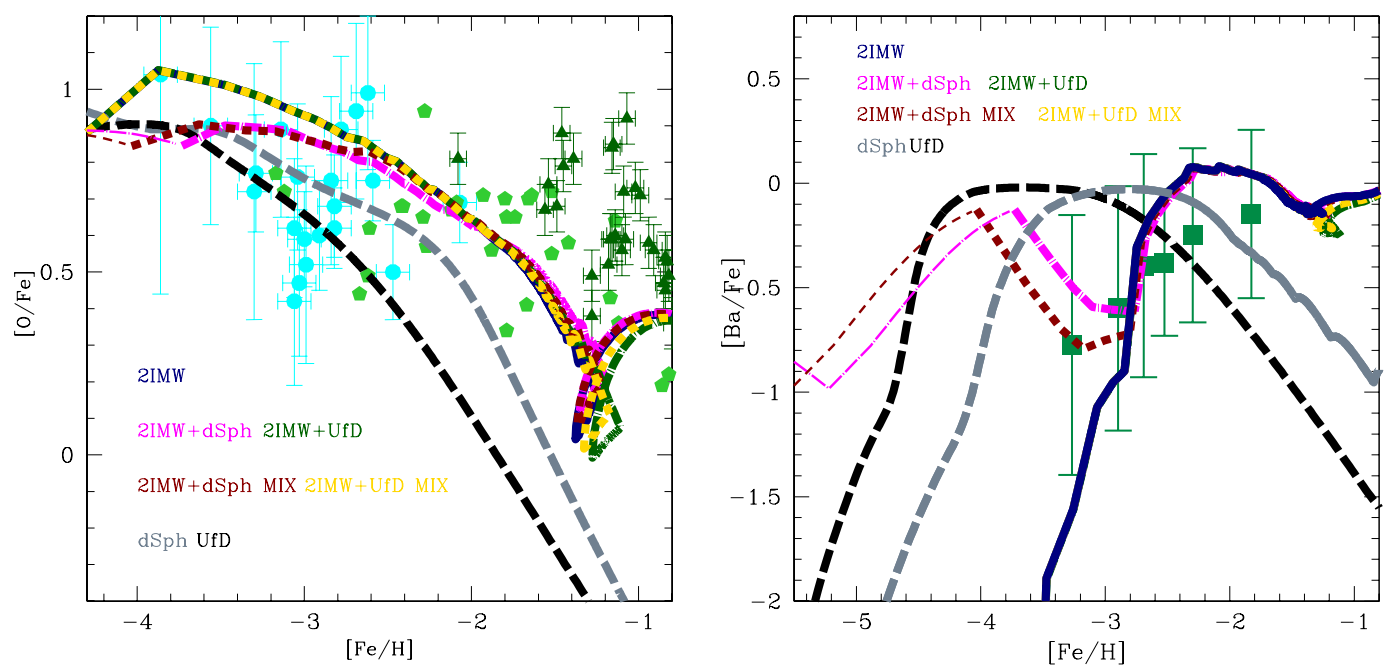

Figure 3: As in Fig. 2 but for the 2IMW model.

model provides now a better agreement with the observed data than the 2IM model, although the predicted $[\mathrm{Ba} / \mathrm{Fe}]$ ratios at $[\mathrm{Fe} / \mathrm{H}]<-3$ dex still lie below the observed data. On the other hand, by assuming an enriched infall from $\mathrm{dSph}$ or UfD galaxies, the predicted $[\mathrm{Ba} / \mathrm{Fe}]$ ratios agree with the observed data also at $[\mathrm{Fe} / \mathrm{H}]<-3 \mathrm{dex}$. In conclusion, in order to reproduce the observed $[\mathrm{Ba} / \mathrm{Fe}]$ ratios over the entire range of $[\mathrm{Fe} / \mathrm{H}]$ abundances, a time-dependent enriched infall in the Galactic halo phase is required. We are aware that for Ba more detailed data are needed, therefore at this stage we cannot draw firm conclusions.

\section{Conclusions}

The main conclusions of Spitoni et al. (2016) can be summarized as follows:

1. the predicted $[\alpha / \mathrm{Fe}]$ versus $[\mathrm{Fe} / \mathrm{H}]$ abundance patterns of UfD and $\mathrm{dSph}$ chemical evolution models deviate substantially from the observed data of the Galactic halo stars only for $[\mathrm{Fe} / \mathrm{H}]$ values larger than - 2 dex; this means that at those metallicities the chemical evolution of the Galactic halo was different than in the satellite galaxies. On the other hand, we notice that for Ba the chemical evolution models of dSphs and UfDs fail to reproduce the observational observed data of the Galactic halo stars over the whole range of $[\mathrm{Fe} / \mathrm{H}]$.

2. Concerning the chemical evolution models for the MW in the presence of enriched gas infall we obtain that: the effects on the $[\alpha / \mathrm{Fe}]$ versus $[\mathrm{Fe} / \mathrm{H}]$ plots depend on the infall time scale for the formation of the halo and the presence of a gas threshold in the star formation. In fact, the most evident effects are present for the model 2IM, characterized by the longest time scale of formation $(0.8 \mathrm{Gyr})$, and the longest period without star formation activity among all models presented here.

3. In general, the enriched infall by itself is not capable to explain the observational spread in the halo data at low $[\mathrm{Fe} / \mathrm{H}]$, in the $[\alpha / \mathrm{Fe}]$ versus $[\mathrm{Fe} / \mathrm{H}]$ plots. Moreover, in the presence of 
an enriched infall we need stars produced in situ in dSph or UfD objects and accreted later to the Galactic halo, to explain the data at lowest $[\mathrm{Fe} / \mathrm{H}]$ values.

4. The optimal element to test different theories of halo formation is barium which is (relatively) easily measured in low-metallicity stars. In fact, we have shown that the predicted $[\mathrm{Ba} / \mathrm{Fe}]$ versus $[\mathrm{Fe} / \mathrm{H}]$ relation in dSphs and UfDs is quite different than in the Galactic halo. Moreover, the $[\mathrm{Ba} / \mathrm{Fe}]$ ratio can be substantially influenced by the assumption of an enriched infall. In particular, the two infall plus outflow model can better reproduce the data in the whole range of $[\mathrm{Fe} / \mathrm{H}]$ abundances, and this is especially true if a time dependent enriched infall during the halo phase is assumed.

\section{References}

[1] Akerman, C. J., Carigi, L., Nissen, P. E., Pettini, M., Asplund, M., 2004, A\&A, 414, 93

[2] Brusadin, G., Matteucci, F., Romano, D., 2013, A\&A, 554, A135

[3] Belokurov, V., Zucker, D. B., Evans, N. W., et al., 2006, ApJ, 647, L111

[4] Brown, T. M., Tumlinson, J., Geha, M., et al., 2012, ApJ, 753, L21

[5] Busso, M., Gallino, R., Lambert, D. L., Travaglio, C., Smith, V. V. 2001, ApJ, 557, 802

[6] Cayrel, R., Depagne, E., Spite, M., et al., 2004, A\&A, 416, 1117

[7] Cescutti, G., François, P., Matteucci, F., Cayrel, R., Spite, M., 2006, A\&A, 448, 557

[8] Fiorentino, G., Bono, G., Monelli, M., et al., 2015, ApJL, 798, L12

[9] François, P., Matteucci, F., Cayrel, R., et al. 2004, A\&A, 421, 613

[10] Frebel, A. 2010, Astron. Nachr., 331, 474

[11] Gratton, R. G., Carretta, E., Desidera, S., Lucatello, S., Mazzei, P., Barbieri, M., 2003, A\&A, 406, 131

[12] Grebel, E. K., 2005, IAU Colloq. 198: Near-fields cosmology with dwarf elliptical galaxies, 1

[13] Kennicutt, R. C., Jr, 1998, ApJ, 498, 541

[14] Koch, A., Feltzing, S., Adén, D., Matteucci, F. 2013, A\&A, 554, A5

[15] Norris, J. E., Gilmore, G., Wyse, R. F. G., et al., 2008, ApJ, 689, L113

[16] Norris J. E., Yong D., Frebel A., Wilkinson M. I., Belokurov V., Zucker D. B., 2010, ApJ, 723, 1632

[17] Romano, D., Karakas, A. I., Tosi, M., Matteucci F., 2010, A\&A, 522, A32

[18] Schmidt, M., 1959, ApJ, 129, 243

[19] Spitoni, E., 2015, MNRAS, 451, 1090

[20] Spitoni, E., Vincenzo, F., Matteucci, F., Romano, D., 2016, MNRAS, 458, 2541

[21] Tollerud, E. J., Bullock, J. S., Strigari, L. E., Willman, B., 2008, ApJ, 688, 277

[22] Vincenzo, F., Matteucci, F., Vattakunnel, S., Lanfranchi, G. A., 2014, MNRAS, 441, 2815

[23] Woosley, S.E., Weaver, T.A. 1995, ApJS, 101, 181

[24] York, D.G., Adelman, J., Anderson, J.E., Jr., et al., 2000, AJ, 120, 1579 